\title{
BIOINFORMATION

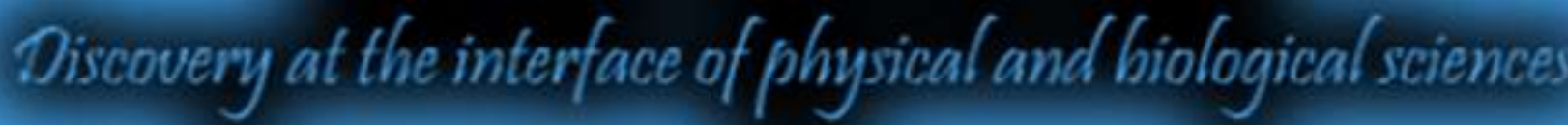

\section{Views and opinion on BDNF as a target for diabetic cognitive dysfunction}

\author{
Allam Appa Rao \\ CR Rao Advanced Institute for Mathematics, Statistics \& Computer Science (AIMSCS), University of Hyderabad Campus, \\ Gachibowli, Prof. CR Rao Road, Hyderabad, Andhra Pradesh, India 500 046; Allam Appa Rao - E-mail: apparaoallam@gmail.com; \\ *Corresponding author
}

Received June 05, 2013; Revised June 17, 2013; Accepted June 17, 2013; Published June 29, 2013

\begin{abstract}
:
Type 2 diabetes mellitus (T2DM) is a known cause of cognitive dysfunction and involves increased risk of dementia. Brain-derived neurotrophic factor (BDNF) is a member of neurotrophic family of nerve growth factors, a key protein in promoting memory, growth and survival of neurons. BDNF is recognized as a metabotrophic factor, a molecule that is involved in Alzheimer's disease (AD) as well as in other neurological disorders. It provides cellular and local regulatory mechanisms for mediating synaptic plasticity. Impaired BDNF signaling can compromise many aspects of brain functions. Studies investigating the relationship between diabetes and BDNF in adults demonstrate that BDNF levels are decreased in T2DM and are regulated in response to plasma levels of glucose. BDNF could serve as biomarker in predicting the development of obesity and T2DM. Thirty-two cavities were predicted to locate the active sites of BDNF for the ligands to bind. The shape of the site was identified by extracting the cavity volume surfaces enclosing regions with highest probability. Different ligands can be chosen for interaction of active sites of BDNF and can be targeted for drug discovery. This review focuses on computational exploitation selectively to deliver BDNF as a drug to appropriate hypothalamic neurons, which can serve as a novel approach in diabetic encephalopathy treatment.
\end{abstract}

Key words: Brain derived neurotrophic factor, BDNF, Diabetes, cognition, computational approach.

\section{Background:}

Type 2 diabetes mellitus (T2DM) is associated with obesity, hypertension, altered lipid profiles, disturbed cortisol control, and low-grade inflammation and found to have cognitive impairment [1]. T2DM patients also have an increased incidence of Alzheimer's disease (AD) and increased incidence of vascular dementia [2]. T2DM has been associated with reduced performance on numerous domains of cognitive function [3]. Both cognitive impairments and structural brain abnormalities have been reported in T2DM, with several studies demonstrating that the hippocampus is specifically affected by the disease [4]. The exact pathophysiology of cognitive dysfunction in T2DM is not completely understood, but it is likely that hyperglycemia, vascular disease, hypoglycemia, and insulin resistance play significant roles [5]. All of these factors have individually been shown to contribute to functional and structural brain changes and may modify the impact of T2DM on brain and cognition. The deleterious effects of diabetes mellitus on the retinal, renal, cardiovascular, and peripheral nervous systems are widely acknowledged [6, 7] offering less attention to the effect of diabetes on cognitive function. Diabetic encephalopathy mainly refers to cognitive injury caused by diabetes, which includes deficits on learning, memory and the ability in executing affairs and solving problems however, its mechanism is unknown.

Disconcertingly, the rapid rise in obesity and T2DM in all age groups might result in a substantial increase in prevalence of diabetes-related cognitive dysfunction. Little of information is available on examination of cognitive dysfunction in patients with diabetes, more needs to be understood about the mechanisms and natural history of this complication in order to develop strategies for prevention and treatment. Deepening 
research on the causable factors in this line can only fetch new medications incessantly.

Neurotrophins are regulatory factors that mediate the differentiation, proliferation and survival of cholinergic, dopaminergic and serotonergic neurons [8]. Brain-derived neurotrophic factor (BDNF) is a member of neurotropic growth factors, which are related to the nerve growth and is a key protein in regulating maintenance, growth and survival of neurons [9]. BDNF is also identified as a key component of hypothalamic pathway that controls body weight and energy homeostasis [10]. It also influences learning and memory [11]. BDNF affects the survival and differentiation of cultured motor neurons, mesencephalic dopaminergic neurons and septal cholinergic neurons [12]. Concerning BDNF, decreased level in the hippocampus is associated with impaired synaptic plasticity, cognitive performances. Increasingly, BDNF has become recognised as a metabotrophic factor [13] with reduced levels reported in several cardio-metabolic diseases, including T2DM.

As a multidisciplinary field, medical informatics draws on a range of disciplines such as computer science, information science and cognitive sciences. Medical informatics can provide important insights into the nature of the processes involved in human-computer interaction and help to improve the application of medical information systems by providing insight into the role that knowledge, memory, and strategies play in a variety of cognitive activities. This review focuses on BDNF protein to be used in treatment of much common diabetic encephalopathy using cognition and computational approach, which can serve as a novel approach in selective delivery of drug.

\section{Diabetes and cognition}

There is a developing body of research identifying the brain as a site of possible complications in T2DM. Both cognitive impairments and structural brain abnormalities have been reported in T2DM demonstrating that the hippocampus is specifically affected by the disease [14]. Decreased cognitive performance has been extensively described among individuals with T2DM, with reduced performance in verbal declarative memory and processing speed being the most consistently reported [15].

T2DM is reported to be contributing to cognitive dysfunction through three indirect mechanisms. Firstly, cognitive dysfunction in patients with T2DM has been correlated to inflammatory markers, and increased inflammation may contribute to the development of AD or macro-vascular disease [16]. Secondly, T2DM could contribute to cognitive dysfunction through the disruption of the hypothalamic-pituitary adrenal axis. Both animals [17] and humans [18] with T2DM have an up-regulation of the hypothalamic-pituitary-adrenal axis, with increased serum cortisol compared with controls. Insulin resistance contributes through the indirect mechanism of upregulating hypothalamic-pituitary-adrenal axis, thereby causing hypercortisolemia related cognitive dysfunction [19]. The third potential mechanism through which insulin resistance may indirectly contribute to cognitive dysfunction is by promoting the formation of senile plaques found in $\mathrm{AD}$ [3].
Cortisol dysregulation, which has been associated with T2DM, had also been shown to impact brain and cognitive function in numerous studies [20]. Modalities to study the effect of T2DM on the brain have evolved over the years, including neurocognitive testing, evoked response potentials, and magnetic resonance imaging [21].

\section{Cognition and BDNF}

BDNF is a molecule that is involved in promoting memory that is reduced in the brain with age and is major factor in AD as well as in other neurological disorders [22]. It has long been considered a drug target for AD [23]. BDNF is a small dimeric protein -that is abundantly and widely expressed in the adult mammalian brain. Various studies have shown possible links between BDNF and conditions, such as diabetes, depression, schizophrenia, obsessive-compulsive disorder, AD, Huntington's disease, Rett syndrome, and dementia, as well as anorexia nervosa and bulimia nervosa [23].

BDNF gene is located on chromosome $11 \mathrm{p} 13$ and is composed of five or more exons, each with its own promoter region allowing for differential mRNA splicing [24]. A de novo paracentric inversion, 46, XX, inv (p12, p15.3), a region encompassing the BDNF locus, was reported with severe hyperphagia, obesity, and complex neurobehavioral phenotypes [25]. Physiological, genetic and neuro-imaging data all favour the hypothesis that BDNF influences neuro-cognitive function. In the brain, BDNF is produced mainly by neurons wherein its levels are increased considerably by electrical activity, and in response to exercise and dietary energy restriction [9]. The transcription factors cyclic AMP response element binding protein (CREB) and NF- $\mathrm{kB}$ induce BDNF expression in response to neuronal activity and metabolic stress [26]. BDNF is released from neurons in an activity dependent manner and binds to a high-affinity receptor tyrosine kinase called trkB that is expressed by neurons and glial cells throughout the nervous system [27].

BDNF can induce its own synthesis and stimulate selective increases in several genes and proteins involved in synaptic plasticity, including synaptotagmin, Homer1a, Arc, and NARP [28]. BDNF can regulate gene expression in the cell body as well as locally at the synapse [29]. This suggests that BDNF provides cellular and local regulatory mechanisms for mediating synaptic plasticity. Thus, impaired BDNF signaling can compromise many aspects of brain function.

\section{BDNF in diabetic cognitive dysfunction /diabetic encephalopathy}

Recent evidence suggests a potential role for brain-derived neurotrophic factor (BDNF) in the relationship between depression and diabetes [30]. High levels of BDNF are found in hippocampal neurons and these are decreased in adult depressed patients [31] but increase with improvements in depressive symptoms, duration of treatment and antidepressant use [32]. Increasingly, BDNF has become recognised as a metabotrophic factor, with reduced levels reported in several cardio-metabolic diseases, including T2DM [13]. Studies investigating the relationship between BDNF and diabetes in adults demonstrate that BDNF levels are decreased in T2DM and are regulated in response to plasma levels of glucose [13]. 
In addition, studies of healthy adults have found that hyperglycaemia inhibits BDNF output from the brain [33].

Concerning BDNF, decreased level in the hippocampus is associated with impaired synaptic plasticity, cognitive performances and mood-related behaviours [34]. Interestingly, interleukin-1b (IL-1 $\beta$ ) modulates memory-induced increase in hippocampal BDNF mRNA expression and signal transduction [35]. Accordingly, elevated cytokine levels observed in the hippocampus of $\mathrm{db} / \mathrm{db}$ mice may contribute to decrease BDNF levels and consequently to impair anxiety-like behaviours and spatial cognitive performances [36].

By examining a large cohort of subjects with or without T2DM in the presence or absence of obesity, Lambert et al. [37] and group concluded that plasma BDNF levels were reduced in patients with T2DM and were inversely associated with fasting plasma glucose levels. Hence, authors proposed that decreased BDNF might be a pathogenic factor linking depression and T2DM. BDNF rapidly regulates energy metabolism in obese diabetic animals, partly through activating the sympathetic nervous system and inducing UCP1 gene expression in BAT [38]. Additionally, both the COMT val158met polymorphism and the BDNF val66met variant appear to influence cognitive function [39], but the specific neuro-cognitive processes involved continue to be a matter of debate. Consequently, such accrued neuro-protective deficits make neurons more vulnerable to injury.

Low levels of BDNF have also been associated with learning/cognitive dysfunction, depression, neurodegenerative conditions, and mortality. BDNF has been studied in various populations; however, the clinical significance of BDNF levels remains unclear in individuals with T2DM [40]. Enhancement of hippocampal BDNF was accompanied by increases in dendritic spine density on the secondary and tertiary dendrites of dentate granule neurons [41]. These studies suggest that diabetes exerts detrimental effects on hippocampal structure, and that this state can be attenuated by increasing energy expenditure and decreasing energy intake.

BDNF as drug target for diabetic cognitive dysfunction through computational approach

Currently, the major drug discovery paradigm for neurodegenerative diseases is based upon high affinity ligands for single disease-specific targets but T2DM mediated diabetic encephalopathy is least probed. Based on the evidences, our group earlier proposed that obesity and T2DM could be disorders of the brain and BDNF could serve as a biomarker in predicting their development [42]. Hence, methods developed selectively to deliver BDNF to appropriate hypothalamic neurons may form a novel approach in treatment. We also investigated three groups of sequences among a series of 12 , with an E-value between 0-12, common to both AD and T2DM: butyrylcholinesterase precursor $\mathrm{K}$ allele (NP 000046.1), acetylcholinesterase isoform E4-E6 precursor (NP 000656.1), and apoptosis-related acetyl-cholinesterase (1B41|A; [43]). The human and mouse data suggest that BDNF mRNA expression peaks in recovery after exercise, while an increased release occurs during exercise [44]. BDNF gene regulation during recovery from exercise serves as the basis for cumulative effects of repeated exercise bouts, eventually leading to detectable increases in BDNF protein content and concomitantly increased potential for BDNF release and neuro-protection in specific brain parts [44].

Our group earlier reported by active site prediction to identify the cavities present in the BDNF protein [23]. Thirty-two cavities are given with the sequence, cavity point and volume of cavity to locate the active sites of BDNF for the ligands with which to bind. It has predicted the shape of the site by extracting the cavity volume surfaces enclosing regions with highest probability. Active site prediction of BDNF is done to identify the locations of ligand binding sites and to predict functional similarities between cavities to estimate the locations of bound ligands. Active site of BDNF is obtained by using active site prediction server. The cavity1 is identified as the cavity highest volume with the following amino acid sequence as the active site RTCEDFAIVKYSQGWLN the cavity points are $5.312 \mathrm{~A}^{\circ}, 26.603 \mathrm{~A}^{\circ}, 20.190 \mathrm{~A}^{\circ}$ and the cavity volume is $1498 \mathrm{~A} 3$. The active site prediction is done to identify the cavities present in the BDNF protein [23]. The different ligands can be chosen for interaction of the active sites of BDNF. In conclusion, the identified active sites of BDNF and the cavities can be targeted for drug discovery.

\section{Conclusion:}

The above reports demonstrate the potential of a new drug discovery paradigm for cognitive dysfunction in T2DM, which can be based upon the requirement that drug candidates be highly effective in multiple, distinct cell culture models of neuro-degeneration. This approach may yield BDNF as very potent, orally active molecule that targets several different pathways that decline in diabetic encephalopathy. Based on the reports available and our research in these lines, we propose BDNF as exceptionally potent neuro-trophic molecule that can prevent the loss of synaptic proteins and cognitive decline in a diabetic encephalopathy.

\section{Acknowledgement:}

The author gratefully acknowledges Department of science and technology (DST) for its financial support through IRHPA schemes vide (L.No: IR/SO/LU/03/2008/1 dated 24-12-2010) and also partial support from DST grants NO: SR/S4/MS: 516/07 dated 21 st April 2008.

\section{References:}

[1] Taki Y et al. Obesity (Silver Spring). 2008119 [PMID: 18223623]

[2] Biessels GJ et al. Lance Neurol. 2008 7: 184 [PMID: 18207116]

[3] Kodl CT et al. Diabetes. 2008 57: 3083 [PMID: 18694971]

[4] Bruehl H et al. Brain Res. 2009 1280: 186 [PMID: 19463794]

[5] Kodl CT et al. Endocr Rev. 2008 29: 494[PMID: 18436709]

[6] Reske-Nielsen E et al. Diabetologia. 1965 1: 233

[7] Lago RM et al. Lancet. 2007 370: 1129 [PMID: 17905165]

[8] Poo M, Nat Rev Neurosci. 2001 2: 24 [PMID: 11253356]

[9] Mattson MP et al. Ageing Res Rev. 2004 3: 445 [PMID: 15541711]

[10] Wisse BE et al. Nat Neurosci. 2003 6: 655 [PMID:12830151]

[11] Tyler WJ et al. Learn Mem. 2002 9: 224 [PMID:12359832]

[12] Henderson CE et al. Nature. 1993 363: 266 [PMID:8487864]

[13] Chaldakov GN et al. Diabetologia. 2007 50: 1781

[14] Gold SM et al. Diabetologia. 2007 50: 711 [PMID: 17334649] 
[15] Awad NE et al. J Clin Exp Neuropsychol. 2004 26: 1044[PMID: 15590460]

[16] Yaffe KJ, Nutr Health Aging. 2006 10: 293 [PMID: 16886099]

[17] Tojo CE et al. Endocr J. 1996 43: 233[ PMID: 9026270]

[18] Lee ZSE et al. Diabetes Care. 1999 22: 1450 [PMID: 10480508]

[19] Hazari MAH et al. Int J Diab Mellitus. 2011. doi:10.1016/j.ijdm.2011.01.001

[20] Starkman MN et al. Biol Psychiatry. 1992 32: 756 [PMID: 1450290]

[21] Akisaki TE et al. Diabetes Metab Res Rev. 2006 22: 376 [ PMID: 16506272]

[22] Tapia-Arancibia E et al. Brain Res Rev. 2008 59: 201 doi: 10.1016/j.brainresrev.2008.07.007. Epub 2008 [PMID:18708092]

[23] Satyavani Get al. Asian J Medic Sci. 2012 4: 8

[24] Jiang XE et al. Neuropsychopharmacology. 2005 30: 1353 [PMID:15770238]

[25] Gray JE et al. Diabetes. 2006 55: 3366 [PMID: 17130481]

[26] Lipsky RH et al. J Neurochem. 2001 78: 254 [PMID: 11461961]

[27] Reichardt LFE et al. Philos Trans R Soc Lond B Biol Sci. 2006 361: 1545 [PMID: 16939974]

[28] Tartaglia NE et al. J Biol Chem. 2001 276: 37585 [PMID: 11483592]

[29] Steward OE et al. Annu Rev Neurosci. 2001 24: 299 [PMID: 11283313]
[30] Korczak DJ et al. Diabetologia. 2011 54: 2483 [PMID: 21789690]

[31] Nestler EJ et al. Neuron. 2002 34: 13 [PMID: 11931738]

[32] Brunoni AR et al. Int J Neuropsychopharmacol. 2008 11: 1169 [PMID: 18752720]

[33] Krabbe KS et al. Diabetologia. 2007 50: 431 [PMID:17151862]

[34] Yamada KE et al. J Pharmacol Sci. 2003 91: 267[PMID: 12719654]

[35] Tong LE et al. Neurobiol Aging. 2008 29: 1380 [PMID: 17467122]

[36] Dinel ALE et al. PLoS One. 2011 6: e24325 [PMID: 21949705]

[37] Lambert GW et al. Diabetologia. 2007 50: 2027 [PMID: 17634920]

[38] Tsuchida AE et al. Int J Obes Relat Metab Disord. 2001 25: 1286 [PMID: 11571589]

[39] Epperson CE et al. Biol Psychiatry. 2012 72: 434 [PMID: 22906753]

[40] Swift DL et al. PLoS One. 2012 7: e42785. doi: 10.1371/journal.pone.0042785. [PMID: 22880108]

[41] Stranahan AM et al. Hippocampus. 2009 19: 951 doi: 10.1002/hipo.20577. [PMID: 19280661]

[42] Rao AA et al. Med Hypotheses. 2008 70: 424 [PMID: 17553627]

[43] Rao AA et al. Lipids Health Dis. 2006 5: 28. [PMID:17096857]

[44] Rasmussen PE et al. Exp Physiol. 2009 94: 1062 [PMID: 19666694]

Edited by $P$ Kangueane

Citation: Rao, Bioinformation 9(11): 551-554 (2013)

License statement: This is an open-access article, which permits unrestricted use, distribution, and reproduction in any medium, for non-commercial purposes, provided the original author and source are credited 